\title{
The Changing Face of Organic Synthesis
}

\author{
Steven V. Ley ${ }^{\star}$ and lan R. Baxendale
}

\begin{abstract}
The article describes the content of the Paul Karrer Lecture given at the University of Zürich on the 20th of June 2007 by Professor Steven V. Ley. The lecture illustrates the work underway within the Chemistry Department at Cambridge to develop microreactors for flow chemistry applications. These modular, small footprint devices are capable of preparing a wide range of compounds including natural products in up to seven synthesis steps. Products can generally be obtained in high yield and purity without conventional work-up methods using a variety of reaction mixer chips and pre-packed flow tubes of immobilised reagents and scavengers.
\end{abstract}

Keywords: Flow-mode synthesis · Microreactors

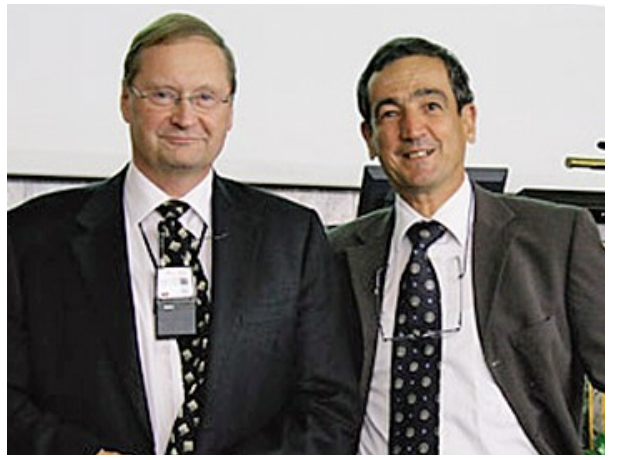

Prof. Steven Ley and Prorektor Prof. Heini Murer on the occasion of the Karrer Lecture
${ }^{\star}$ Correspondence: Prof. S. V. Ley

Department of Chemistry

University of Cambridge

Lensfield Road, Cambridge

CB2 1EW, UK

E-mail: svl1000@cam.ac.uk
The art and craft of assembling functional molecules from small chemical building blocks has reached an impressive level of sophistication. Today, by harnessing the power of modern synthesis methods, one can access a truly staggering array of compounds that display structural or beneficial properties and function. These range from healing drugs to compounds that guarantee the quality and quantity of the food supply to all the modern needs of society ranging from paints, pigments and dyestuffs to plastics and polymers of all kinds.

Yet despite the obvious success of chemical synthesis we also recognise its limitations and the need for improvement. This is especially true if we wish to move to more sustainable chemical practices, as we must, if we are to protect our natural resources. In order to safely respond to the needs of improved productivity and efficiently we must embrace these new challenges and opportunities and explore alternative approaches to compound synthesis. The current costs, scale-up issues, lack of reproducibility, manpower wastage through repetitive and or routine tasks are unacceptable; therefore change is inevitable.

Fortunately, the modern chemist is responding and we now have a range of new tools available for more effective synthesis of molecules. ${ }^{[1]}$ These maybe biochemical such as gene shuffling methods, or exploiting multi-enzymes in synthesis as well as using directed evolution techniques. They may involve new devices such as microarrays, mini-reactors, diagnostic reporter systems and a plethora of opportunities created by our increasing ability to exploit advances in nanoscience. We benefit enormously by the use of automation, the integration of Design of Experiment optimisation software and by informatics and database mining. Similarly, no chemist now works without computational tools for shape and cluster analysis, compound design or diversity algorithms.

There are also many chemical tools that are influencing the way we work today. These may be parallel synthesis tools or come from opportunities created by fast serial processing using focussed microwave methods or microfluidic devices. Polymersupported reagents, scavengers and catch and release techniques are also having their impact on the way synthesis programmes are conducted. Moreover, as we pointed out a number of years ago, when these methods are combined with flow chemistry devices, enormous opportunities arise. ${ }^{[2]}$ These principles and ideas form the basis of this article. More information and details can be found on our website: http://leyitc.ch.cam. $a c . u k /$ and in our recent reviews on the subject. ${ }^{[3-7]}$ Although over the years we have published extensively on the application of supported systems, we illustrate here the power of these methods with the preparation of the natural product carpanone ${ }^{[8]}$ (Scheme 1). This proceeds from a simple starting material, sesamol, and leads to the structurally more complex naturally occurring material in $66 \%$ overall yield in just four steps through six unit operations involving five supported reagents and scavengers. The whole process gives a high purity product without recourse to conventional work-up protocols such as chromatography, water washes, distillation or crystallisation. Similarly, more complex targets can be addressed using these methods, such as drug substances ${ }^{[9,10]}$ or natural products like the plicamines ${ }^{[11]}$ and the epothilones. ${ }^{[12]}$ This chemistry forms 
the basis for our approach to flow-mode synthesis. One can easily envisage, that by simply packing a glass column or cartridge with an immobilised reagent or scavenger for impurity capture, one could set up a device for automated multi-step synthesis using modular and readily reconfigured components. In a typical flow arrangement reactants and solvents can be pumped through microfluidic serpent channelled reactor chips or through prepacked supported reagent columns, the product stream can be analysed, recycled if necessary or collected. In certain configurations the output stream can be directed into microfluidic biological flow assays or indeed into some other functional read out device. This concept is very attractive for high throughput reactions or reagent screening processes. The act of immobilisation means that it is easy to interact physically with the packed reagent or scavenger cartridge using, for example, heat, oscillation, cooling, microwave, ultraviolet or ultrasound methods to bring about a desired chemical transformation (Scheme 2).

Many pumps, chips, control valves, reagent input devices, column heater and product collection units are now commercially available. Our approach has been to select and integrate these pieces of equipment as required by the chemistry. This ensures we maintain maximum flexibility and create small footprint modular arrangements, which are readily and rapidly reconfigurable. Moreover, the apparatus can be set up to deliver compound collections, or, by using scale up and scale out techniques, significant quantities of material. Below we illustrate, with selected examples, how these methods can be employed to prepare useful building blocks, to achieve important transformations, and to develop concepts for the construction of natural products by multi-step processes in the flow-mode.

Oxazoles are well established as heterocyclic core structures of importance in both pharmaceutical and agrochemical products. Accordingly, by reacting an acid chloride with an isocyanide input on a $1 \mathrm{ml}$ reactor chip, followed sequentially by passage of the flow stream through an immobilised base to effect cyclisation, a crude stream of the oxazole product can be generated. A prepacked tube containing a benzyl amine resin (Quadrapure BZA) was then used to scavenge any excess acid chloride, yielding the purified 4,5-oxazole products in excellent yield (Scheme 3). While some thirty compounds were prepared in the first instance by this method in 50-200 mg quantities, several were deemed to be worthy of further development. Consequently, by simply replacing the reagent and scavenging columns with larger pre-packed tubes, we were able to deliver $10 \mathrm{~g}$ quantities of any of the selected materials in an overnight run. The apparatus for this synthesis is shown in Fig. 1 and described in detail in the supplementary material in the resulting publication. ${ }^{[13]}$

During other studies designed to show that either unstable or potentially hazardous compounds can be manipulated safely in the flow-mode, we reacted various azides with acetylenes in dichloromethane to give the corresponding triazole products cleanly and in high yield (Scheme 4). Both the azide and the acetylene inputs may also be generated by flow chemistry methods and telescoped into these syntheses. After mixing the inputs (azide/acetylene) from preloaded reagent loops on a reaction chip, the flow stream was subsequently passed through an Amberlyst 21/CuI reagent plug to affect the cycloaddition reaction. The exit stream was subjected to scavenging with a Quadrapure thiourea resin cartridge to remove any leached copper arising from the catalyst. Subsequently passage through an additional phosphine pre-packed tube to scavenge any excess azide gave the triazole product (Scheme 4). Using this reaction scheme, a small collection of 14 triazoles was prepared in yields ranging from

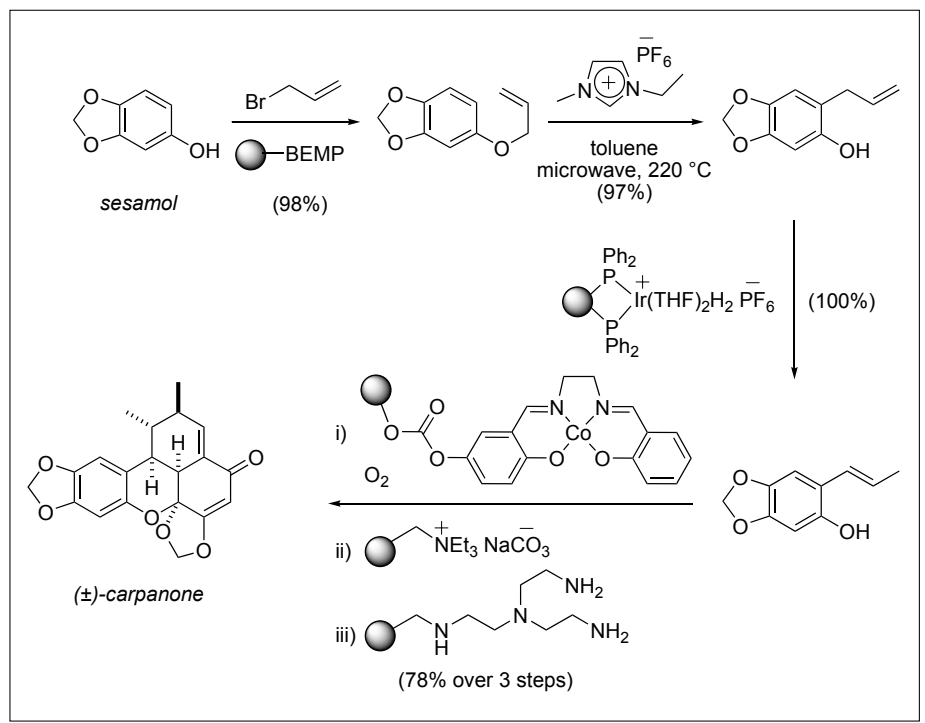

Scheme 1.

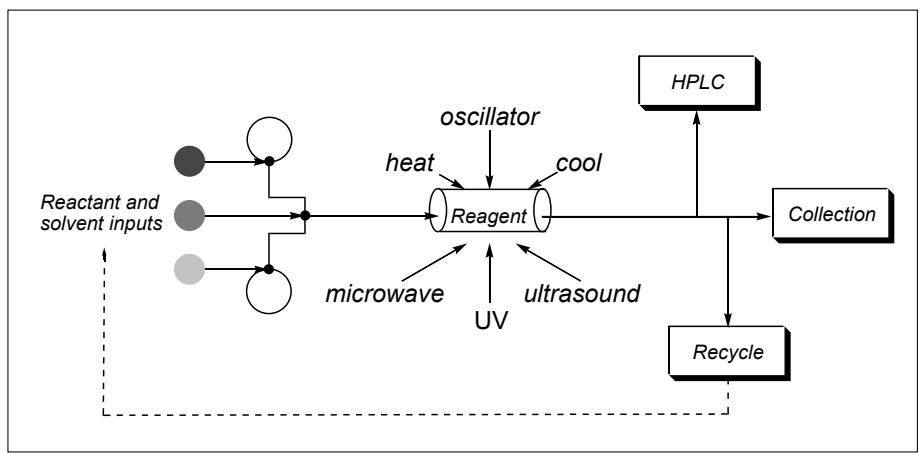

Scheme 2.

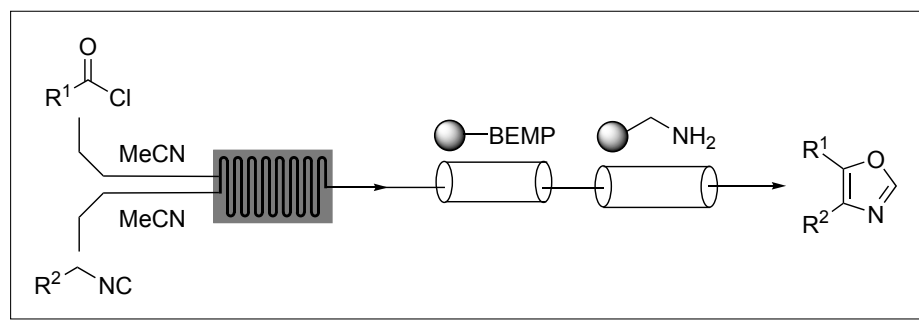

Scheme 3

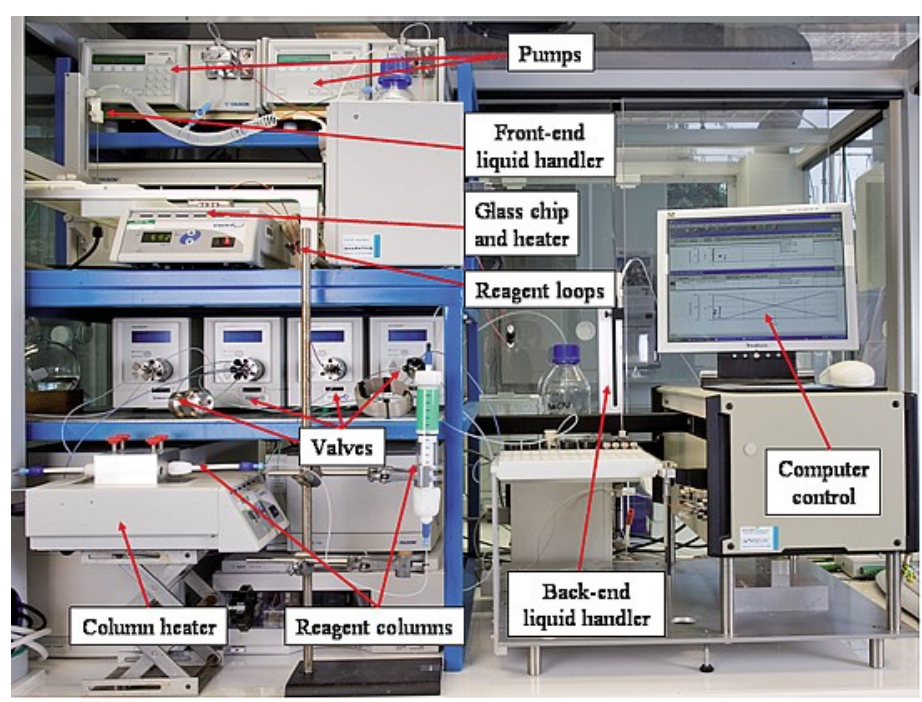

Fig. 1. Modular flow equipment suitable for multi-step synthesis 
75-98\% and in purities all greater than $95 \% .{ }^{[14]}$ No attempt was made to scale these reactions above $1.5 \mathrm{~g}$, however this would be possible by incorporation of larger flow tubes. Also we noticed that since oxygen was easily excluded in the flow system no products arising from Glaser coupling reactions were observed.

Another reaction that is considered to be of strategic importance and yet is not extensively used owing to safety issues is, of course, the Curtius reaction. Given that in flow-mode any highly reactive and unstable intermediates are generated in a constant stream, in small quantities, then immediately consumed in a subsequent reaction, this concept has significant consequences and potential applications for organic synthesis. We have shown, therefore, that we can perform Curtius reactions by reacting acids with diphenylphosphoryl azide (DPPA) in the presence of triethylamine and capturing the intermediate isocyanates with a nucleophile by passage through a convection flow coil (CFC) mounted on a Vapourtec R4 heater unit (Fig. 2). On heating at $120^{\circ} \mathrm{C}$, the initially formed acyl azide (a potentially very hazardous component in batch mode) safely rearranges with loss of nitrogen to give the isocyanate intermediate, which is

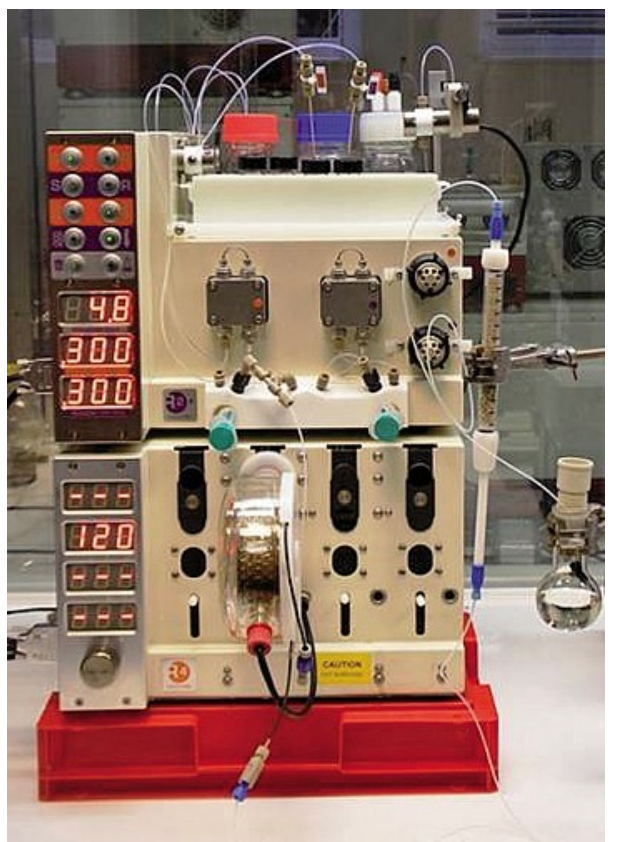

Fig. 2. Vapourtech R2+R4 flow reactor trapped by an in situ nucleophile to give the corresponding product. Passage of the flow stream through a mixed acid-base scavenger bed ensures the formation of a clean product (Scheme 5). For some compounds, however, we found that selective scavenging was necessary to afford the pure product owing to the polar or basic nature of the materials that were being generated (Fig. 3). We have also shown recently that acid chlorides are excellent precursors for Curtius reactions in flow by delivering the necessary azide component from an ion exchange tetra-alkylammonium monolithic support, again using the $\mathrm{R} 2+\mathrm{R} 4$ flow reaction platform.

By using these devices we have also demonstrated that the formation of peptide bonds can be effectively achieved using preloaded HOBt cartridges to activate the appropriate amino acids. ${ }^{[15]}$ This work also led to the development of the use of the $\mathrm{H}$ Cube (Fig. 4), a flow hydrogenation unit, for the deprotection, by hydrogenolysis, of intermediate coupled peptides. ${ }^{[16,17]} \mathrm{We}$ had previously described the use of the HCube for the efficient reduction of imines to amines in flow-mode, which allowed us to make compounds more rapidly than by using conventional methods. ${ }^{[18]}$ Once we had knowledge of these simple reactions, we were able to move to more demanding flow chemistry sequences such as those used in complex natural product syntheses. In the first of these examples, we showed that ferulic acid could be activated by capture onto an HOBt column. ${ }^{[19]}$ Once activated, $p$-hydroxyphenethylamine was introduced to the flow stream to give the coupled amide product. The amide was then subjected to oxida-

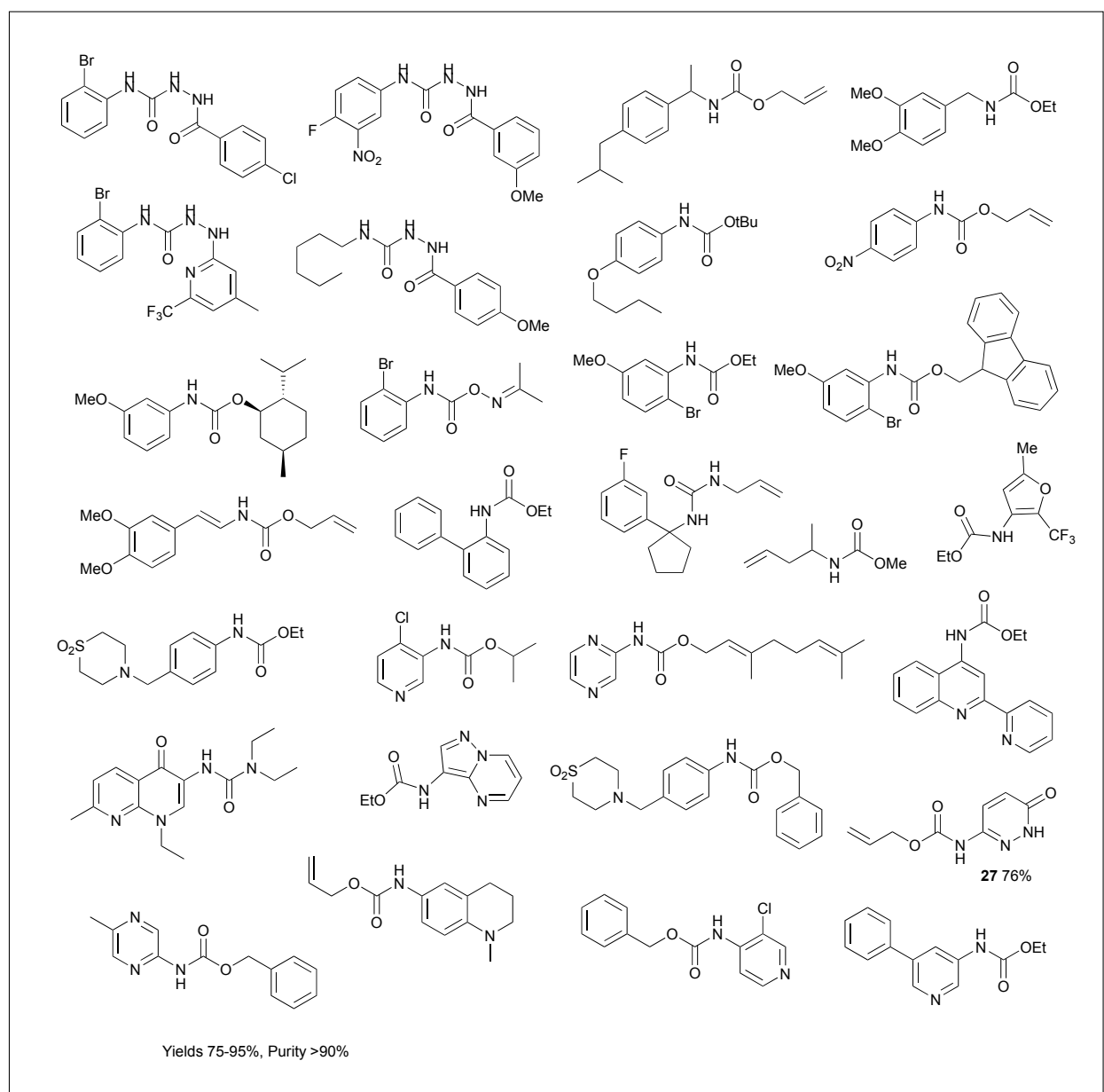

Fig. 3. Collections of Curtius products

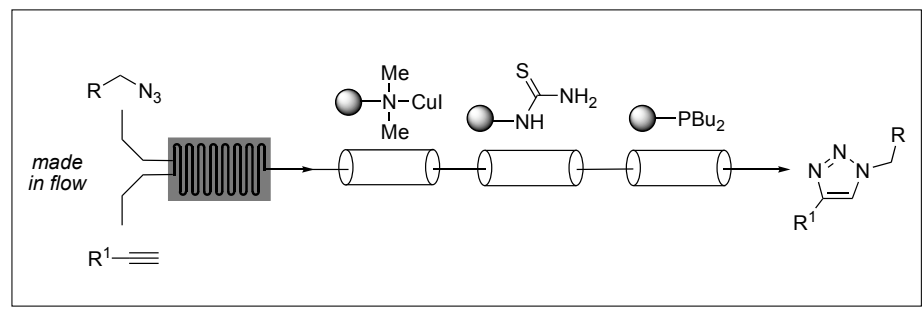

Scheme 4.

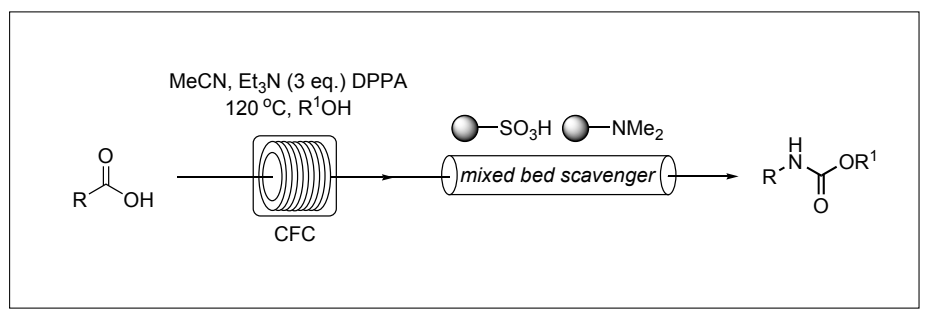

Scheme 5. 
tive dimerisation to give the natural product grossamide in excellent $91 \%$ isolated yield, by using an immobilised horseradish peroxidase II pre-packed flow column to affect oxidative dimerisation (Scheme 6). The use of immobilised enzymes in flow systems is particularly attractive for synthesis since by the judicious use of flow rate control, one can determine the chemoselectivity of the reaction process, since the products of the enzyme reaction can pass rapidly into a benign environment on exiting the flow tube and thereby do not undergo any further unprofitable biotransformation.

In an effort to extend our capabilities in flow chemistry, we have examined another natural product synthesis whereby, a seven-step sequence of reactions was devised to prepare the alkaloid oxomaritidine ${ }^{[19}$ (Scheme 7). This work brings together a

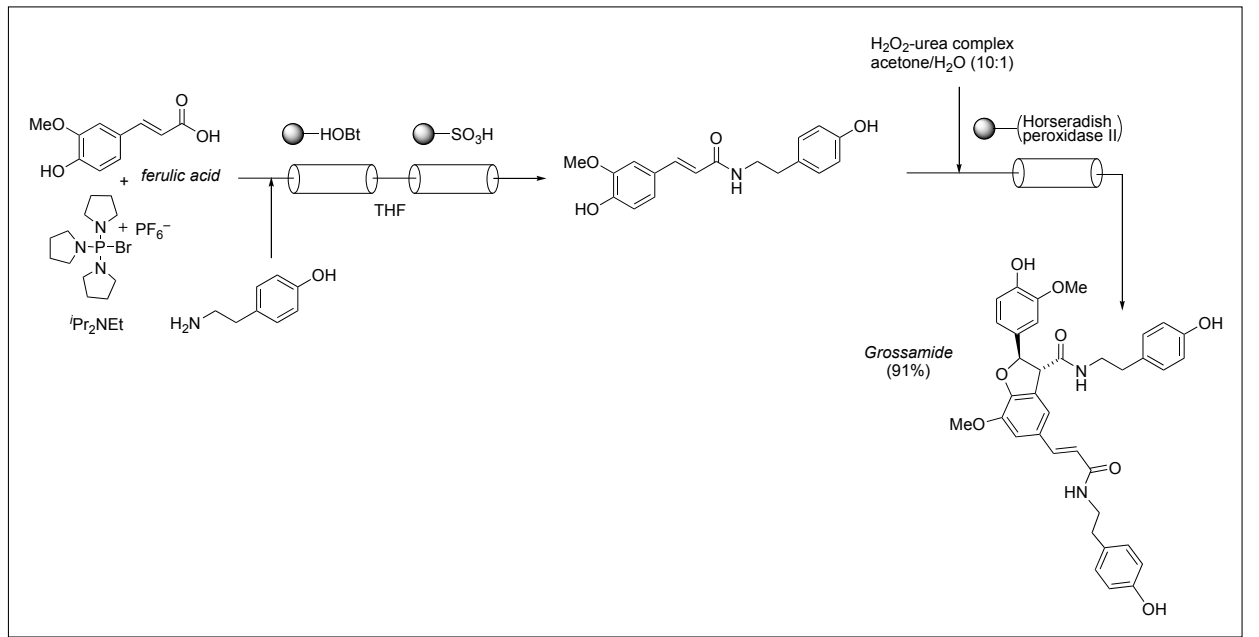

Scheme 6.

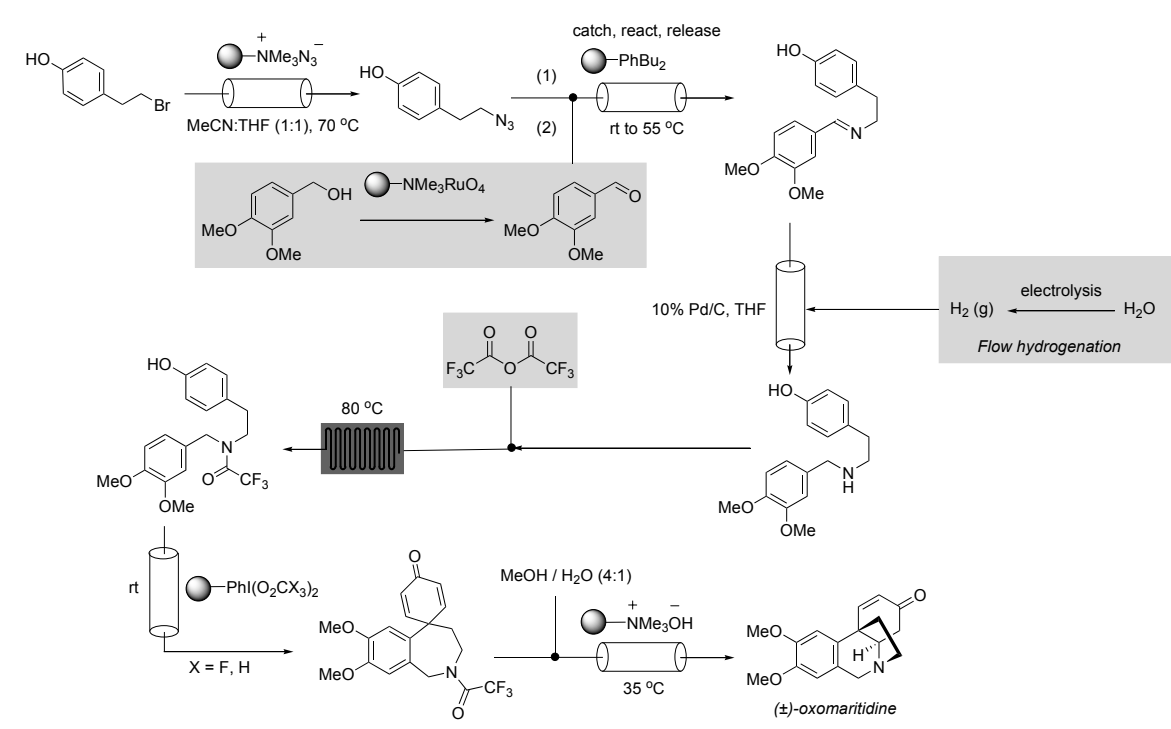

number of beneficial elements under the flow reaction conditions. Firstly, a potentially hazardous azide building block was prepared in situ and then used immediately as an in-line input to generate an immobilised phosphoryl imine reagent. This was subsequently used in an aza-Wittig process by coupling with an aldehyde, itself also generated in a separate flow tube arrangement. The resulting coupled imine was then reduced by application of the $\mathrm{H}-\mathrm{Cube}$ technology described above. Next, the solvent (THF) was removed from the amine product by the use of the Biotage V10 evaporator (Fig. 5) and changed to dichloromethane for the remaining sequence of reactions. These involved protection as the trifluoroacetate through reaction with trifluoroacetic anhydride using an in-line reaction chip, followed by oxidation to the spirodienone using an immobilised hypervalent iodonium ditrifluoroacetate. ${ }^{[20]}$ Finally, mixing the exiting reagent stream with aqueous methanol and passage through an ammonium hydroxide resin caused deprotection and concomitant cyclisation to the natural product oxomaritidine (Scheme 7). This whole sequence of reactions progresses in only five to six hours compared to the normal four days for a conventional synthesis of the molecule. This shortening of the synthesis time by operating in the flowmode is achieved by eliminating typical unit operations such as solvent extractions, water washes, drying and chromatography; common to the traditional approach. Moreover, by releasing skilled operator time in this way, more productive pursuits such as route planning and studying new reactions and processes are possible.

One of the limitations, however, of using these systems is that not all immobilised reagents display the reactivity patterns that we ideally desire. While this loss of reactivity can be compensated for by microwave or ultrasound methods, alternative approaches are necessary. ${ }^{[21]}$ A concept that we have studied therefore involves the development of reagents (or substrates) that contain a chemical tag. Following an 'in solution' phase flow re-

Scheme 7.

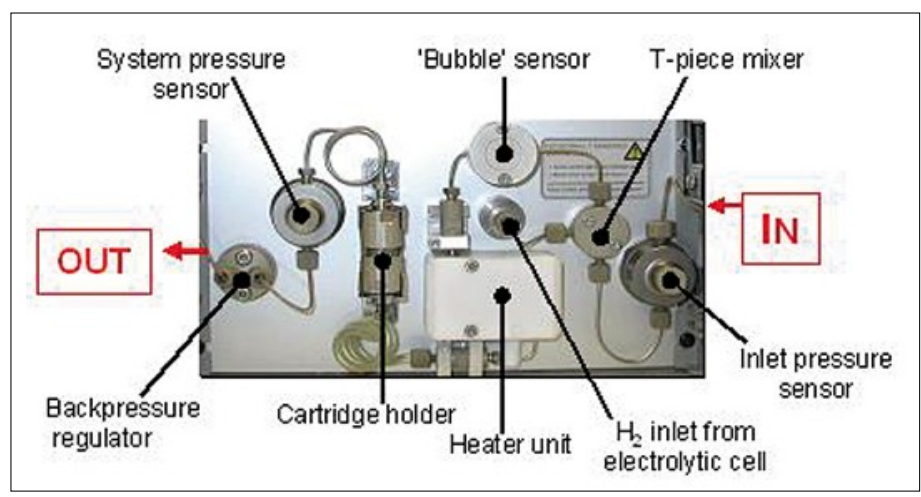

Fig. 4. The Thales $\mathrm{H}$-cube flow hydrogenator

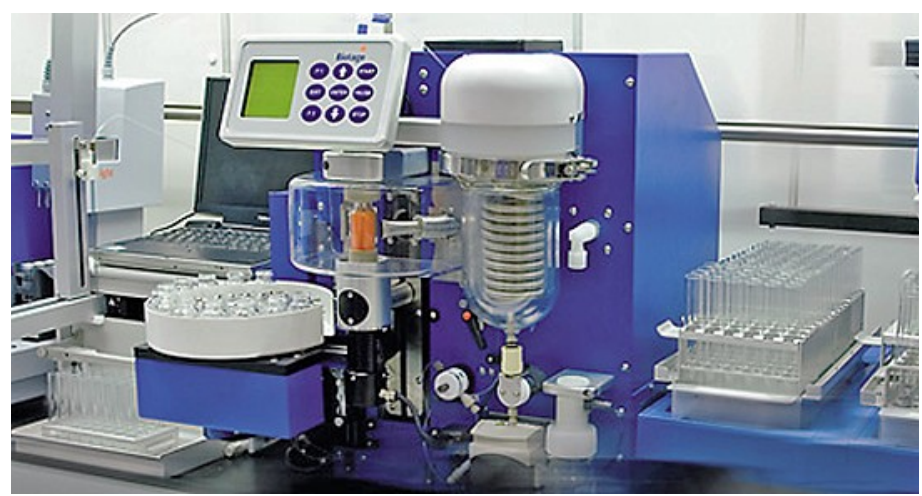

Fig. 5. Biotage V10 evaporator 

switched' by the use of an immobilised complementary chemical process that recognises the tag and binds it selectively to remove the spent reagent from the flow stream. We have used this concept very effectively in the preparation of guanidines, which are notoriously difficult to obtain in very pure forms. In this work, masked acid tagged phosphines were employed. [22] However in this article, by way of a simpler example to illustrate the process, we have devised a bipyridine tagged carbodiimide reagent to affect coupling of amines and acids to generate amides.[23] In this particular example of the phase switch process, the spent reagent (the urea), and the excess reagent are both removed by phase-switch passage through a supported copper species that selectively binds to the bipyridine component to form a complex, thereby removing all these im- action, the spent reagent can be 'phase-

purities from the main amide product reaction stream (Scheme 8).

During recent work on micro and mesoreactors for various flow chemistry applications, we have developed two new systems, which deserve further comment. The first of these is the manufacture and use of plastic microcapillary flow discs (MFDs) for scale-up and scale-out applications. ${ }^{[2]}$ The MFDs are made via a gas entrained polymer extrusion process, whereby a polymer melt is extruded over a series of injectors in a rectangular die so as to produce a continuous polymer tape containing microcapillary channels, the number of which depend on the injectors; normally nineteen (Fig. 6) The tape can then be coiled to produce single or doubly spiralled discs that can be used as a microreactor when reactants and solvents are pumped through the channels. The channels are typically between 180 and $200 \mu \mathrm{m}$ in diameter, with the length of a

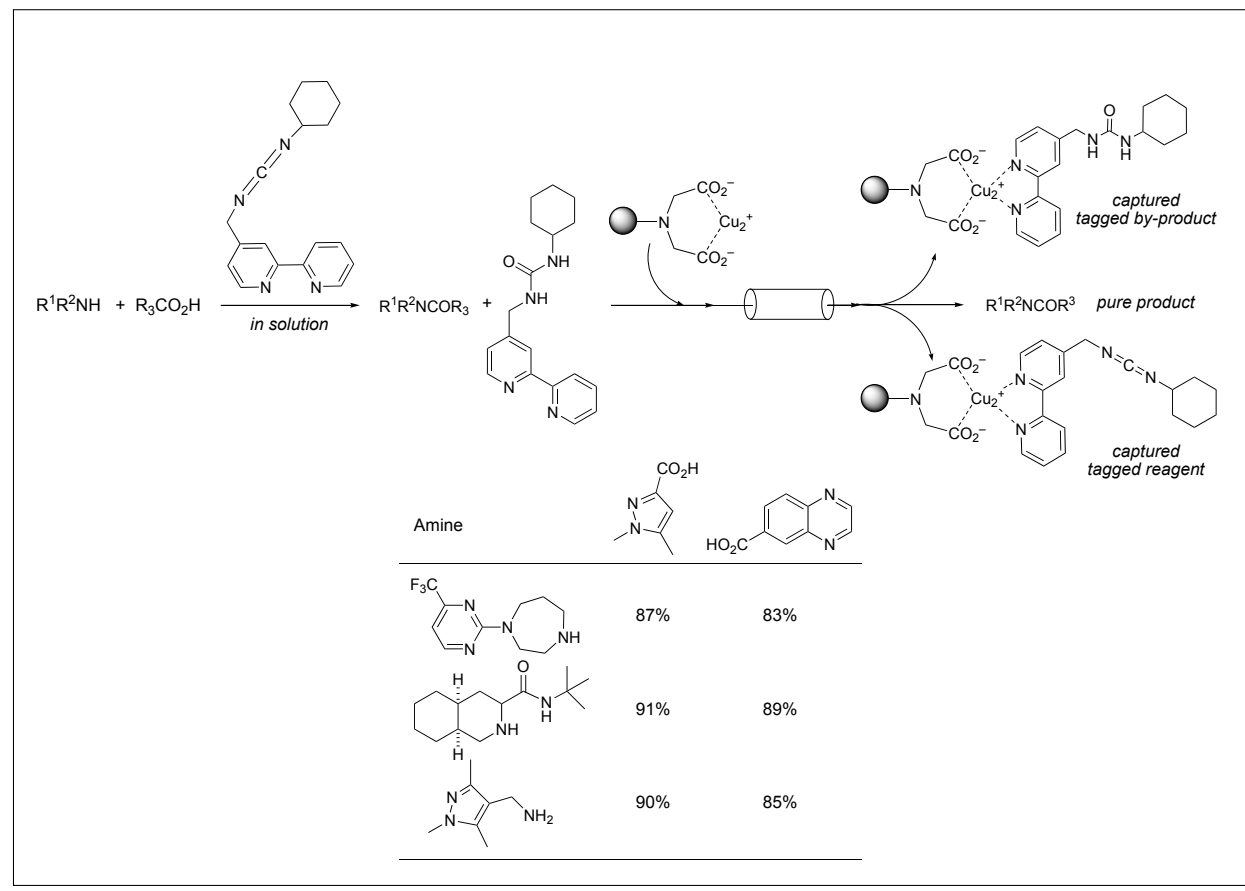

Scheme 8.

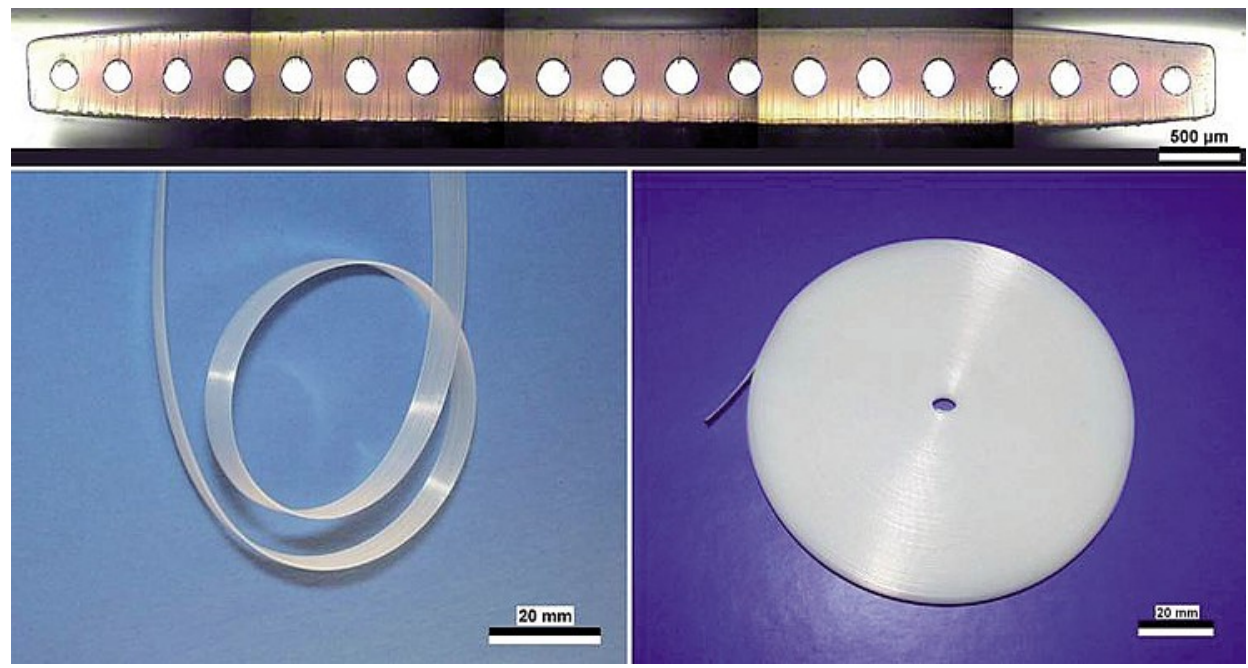

Fig. 6. Microcapillary flow discs reactor coil being between 5 and $40 \mathrm{~m}$. The compact arrangement and the opportunity for recycling make these reactors ideal for scale-out operation. Indeed, a stack of eight of these MFDs in parallel can be readily assembled and connections made to appropriate pumping systems (Fig. 7). Using this arrangement we have performed a simple Diels-Alder reaction to generate, in continuous flow-mode, around $4 \mathrm{~kg}$ of product per day (Scheme 9). Several other chemistries, such as heterocyclic ring formation or alkylation, have also been performed using this particular reactor set up, which further demonstrates its reaction scope. The device is very practical and operates at low cost. The discs allow for fairly rapid heat transfer so that one can accommodate exothermic processes or alternatively, if necessary, they can be externally heated. The system is also compatible with microwave irradiation, consequently it can be readily incorporated into various flow microwave arrangements.

In another project we have developed and evaluated monolithic flow reactor cartridges. ${ }^{25]}$ These were designed to overcome some of the limitations of spherical polymer bead supported reagents in packed flow tubes. These problems include poor fluid dynamics, heat transfer problems and packing and channelling issues. By using a monolithic flow-tube containing a single functionalised macroporous polymer, many of these problems can be overcome. For example, we have prepared monolithic macroporous reactors, using a procedure similar to that described by Frechet and Svec ${ }^{[26]}$ (Fig. 8). This material was then derivatised with palladium nanoparticles to afford a column capable of performing Heck cross-

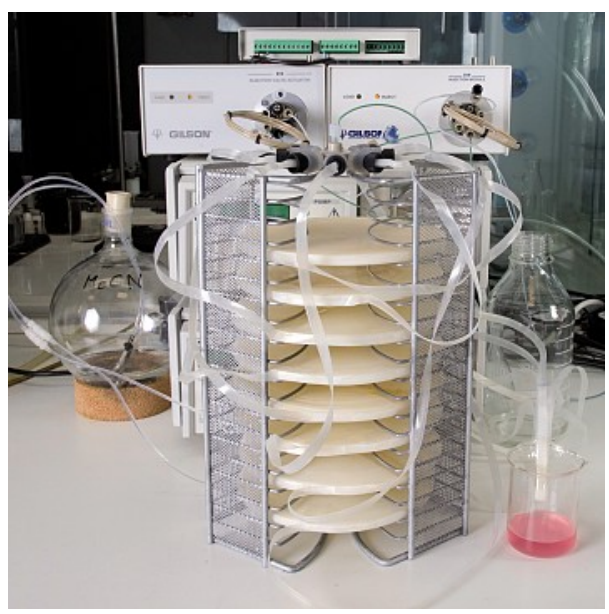

Fig. 7. Scale out mode of operation using microcapillary flow discs

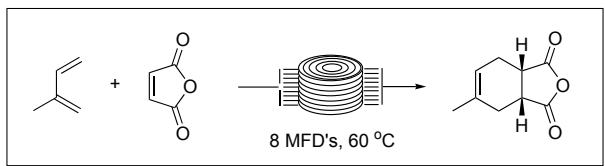

Scheme 9. 
coupling reactions. Pleasingly, these reactions proceeded in excellent yield and purity, and where Pd leaching was observed, simply placing a second metal scavenger column in-line eliminated this problem. Consequently, using a cartridge of Quadrapure TU resin as a scavenger, the ICP-MS analyses showed Pd levels in the product to be below 5 ppm. Importantly, these monolithic reactor cartridges could be reused at least twenty-five times without degradation or affecting the product yields. Also in this work we were able to use ethanol as solvent at $120^{\circ} \mathrm{C}$, since the flow reactor system readily accommodates the additional pressure that is generated. This is achieved by the incorporation of an in-line 100 psi back pressure regulator (Scheme 10). Using ethanol as solvent as opposed to DMF also greatly facilitates the product work up. These monolithic systems are also ideal for smaller scale applications, owing to the higher surface areas and general reproducibility of the flow tubes.

As discussed above, flow microwave units should also be of interest to the synthesis chemist. We have, therefore, designed a U-tube arrangement whereby the central tube may be packed with an immobilised reagent to affect a chemical reaction by selectively absorbing microwave energy. ${ }^{[27]}$ An external cooling jacket is also incorporated into the device to simultaneously moderate the central U-tube temperature (Fig. 9). This arrangement often affords higher yields and higher purity products then are achieved by conventional heating in batch mode. The whole unit is designed to fit into the microwave cavity of commercial machines and is pumped externally using cheap HPLC systems. In-line regulators control the pressure of the apparatus. In one particular application, using commercially available $\mathrm{Pd}$ EnCat catalyst, which is an encapsulated palladium catalyst in polyurea, we showed that the system was particularly effective at carrying out Suzuki cross-coupling reactions in the flow-mode using this external cooling protocol (Scheme 11). By way of a direct comparison, in the experiments we studied, the reactions could be seen to proceed in higher yield and purity than in batch mode. Moreover, a single charge of catalyst was effective for up to 20 different chemical inputs. Alternatively, the device could be used preparatively to generate up to 10 $\mathrm{g}$ of product in a continuous flow process. The exiting flow stream was also subjected to scavenger columns to afford the clean, coupled materials with ICP-MS levels for Pd below 10 ppm. Interestingly, multiple U-tube devices can also be formulated to conduct different chemical processes in the same machine at the same time.

In a related study we have developed a low-cost flow microwave unit which consists of a fluorinated polymer flow tube wound around a central Teflon core fitted with a dummy pressure cap typical of microwave reaction vessels ${ }^{[28]}$ (Fig. 10). The whole unit fits into the cavity of commercial microwave equipment. Once again, external HPLC pumps are used to drive the system and using standard 100
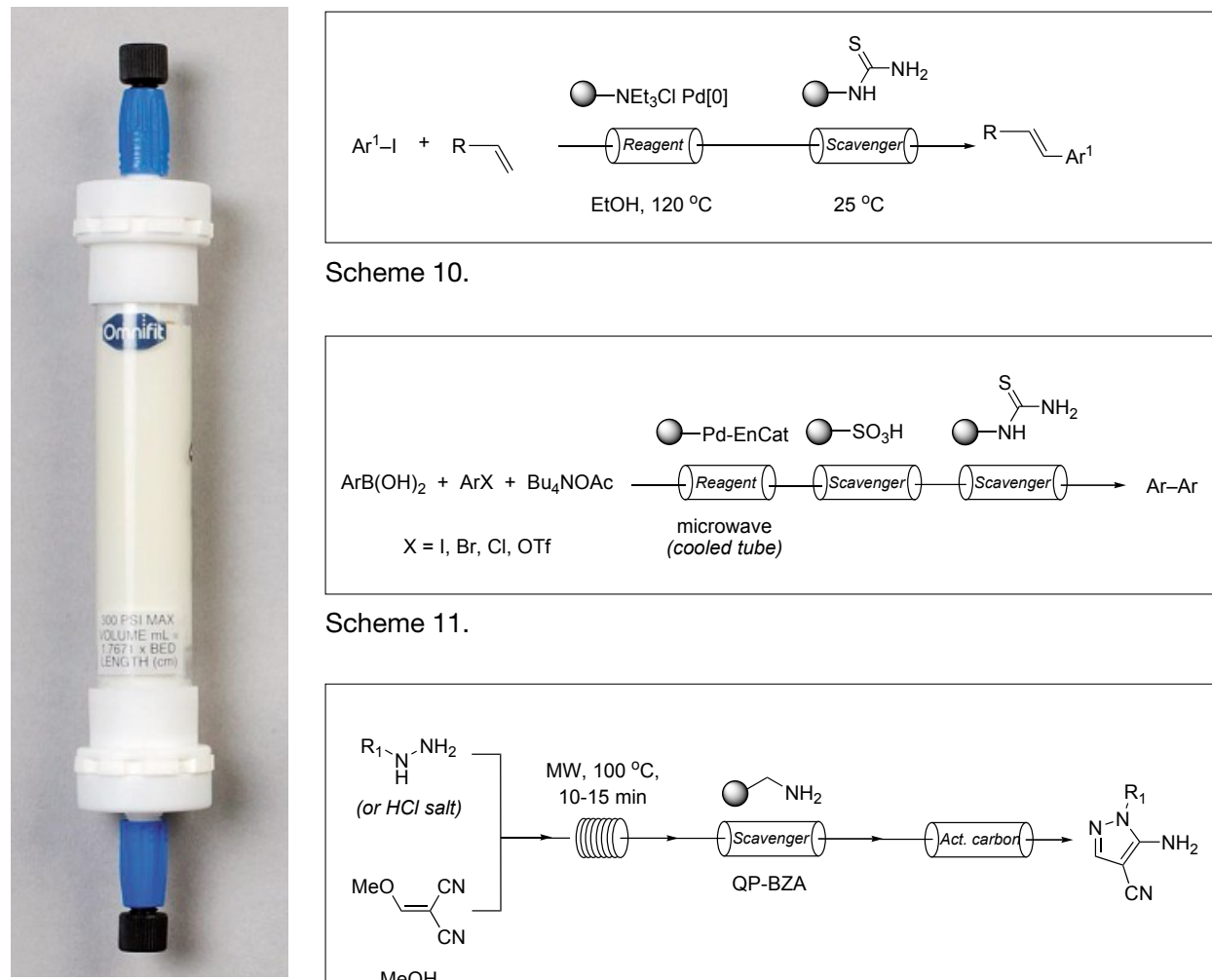

Scheme 10 .

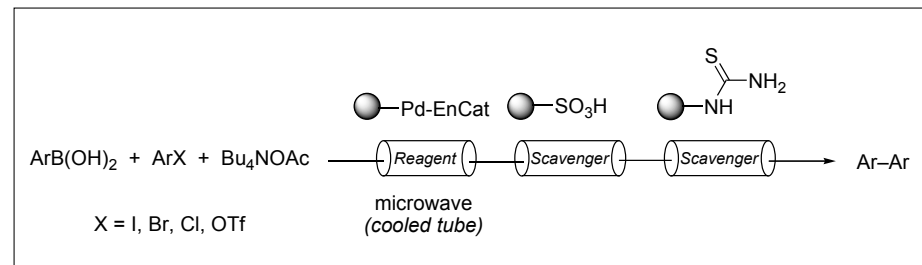

Scheme 11

Fig. 8.

Monolithic flow tube

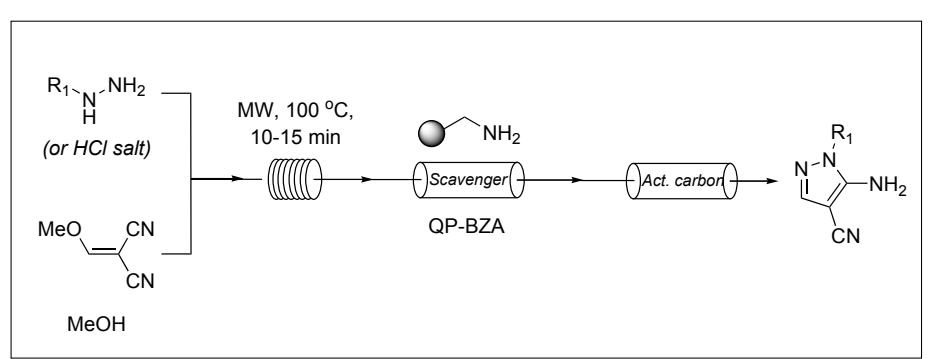

Scheme 12.

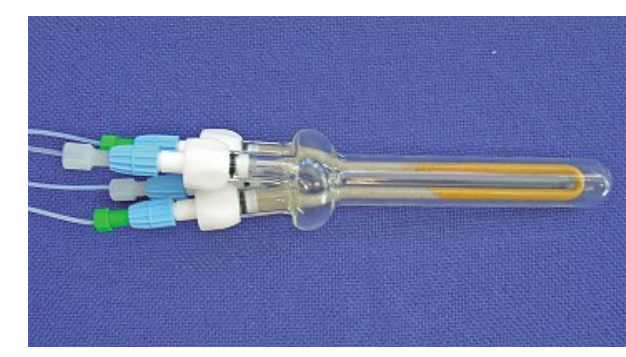

Fig. 9. Cooled microwave flow tube

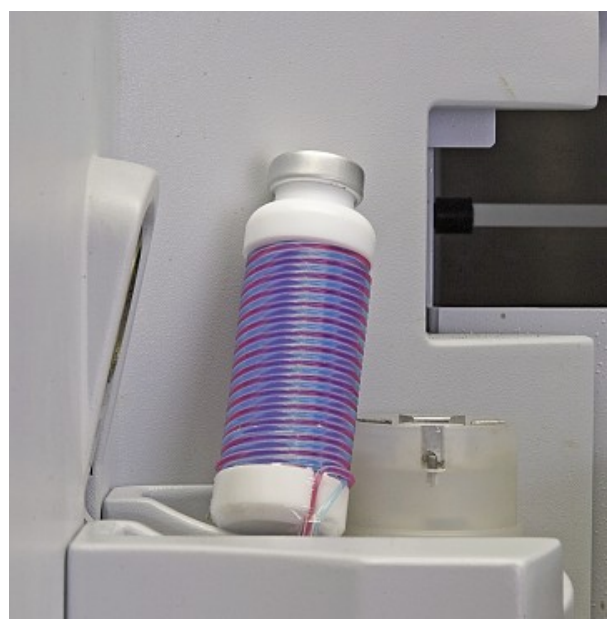

Fig. 10. Microwave flow chemistry

psi pressure regulators to control pressure. The central Teflon spigots are easily re-wrapped should blockages occur, and by varying the length and diameter of the tubing, or wrapping with multiple lengths of tubing we can accommodate many different reactions and flow rates within the same device.

By way of illustration of these particular microwave flow coils, we have shown that by using simple chemical inputs such as hydrazines, when these are reacted with ethoxymethylene malononitrile, one can obtain useful quantities of 5-amino4-cyanopyrazoles as products (Scheme 12). These compounds are important building blocks for many pharmaceutical drug applications. The whole flow system also incorporates a benzylamine scavenger (Quadrapure BZA) and an activated carbon column to ensure high purity of the final product output. In one example we showed that up to $250 \mathrm{~g}$ of compound could be obtained within a three-hour reaction period. Clearly, many other reactions should be possible using these readily adapted and cheap devices.

We recognise we have not been alone in our studies on flow chemistry, therefore we also list some key work of others who have made seminal contributions to the area. ${ }^{29-44]}$ However, because of the very nature of this article, which reviews the 2007 Karrer lecture, this list must be highly selective and we apologise to the authors whose work has not been included. 
We believe the whole area of flow chemistry and the use of microreactors is poised to have a very significant impact on how modern synthesis will be conducted in the future. Nevertheless, while these methods constitute a change in technology, they represent a massive change in philosophy. One should not, therefore, underestimate how much more development will be needed to provide truly robust flow chemistry platforms that will find their way routinely into our laboratory environments. The devices themselves are purely servants to synthesis and the quality of the output will still depend on the thinking and the skill of the individual operators. However, the initial benefits that our research group has observed using these methods have been significant. The general adaptability and ease of scaling-up chemical processes in a 24/7 working environment has been especially attractive. Similarly the waste material per head generated by workers in our flow laboratory is approximately one tenth of that produced in our conventional synthesis laboratory. As we move forward we can anticipate many new opportunities that will include fast serial reaction and reagent profiling. Also the ability to make and immediately screen compounds for biological activity and progress to multi-step syntheses of complex architectures using feedback loops and data evaluation protocols, much faster reagent and compound optimization will be possible. The future of these concepts is both assured and exciting.

\section{Received: February 20, 2008}

[1] S. V. Ley, I. R. Baxendale, Nature Reviews Drug Discovery 2002, 1, 573.

[2] S. V. Ley, I. R. Baxendale, R. N. Bream, P. S. Jackson, A. G. Leach, D. A. Longbottom, M. Nesi, J. S. Scott, R. I. Storer, S. J. Taylor, J. Chem. Soc., Perkin Trans. 1 2000, 3815.

[3] I. R. Baxendale, S. V. Ley, 'New Adventures to Efficient Chemical Synthesis: Emerging Technologies', Symposium Proceeding 06.3, Eds P. H. Seeberger, T. Blume. Springer, Berlin Heidelburg, ISBN 978-3-540-70848-3, 2007, 3, p 151.

[4] S. V. Ley, I. R. Baxendale, R. M. Myers, in 'Comprehensive Medicinal Chemistry
II, Vol. 3, Drug Discovery Technologies', Eds. D. J. Triggle, J. B. Taylor, Elsevier, Oxford, ISBN: 0080445136, 2006, p 791.

[5] S. V. Ley, I. R. Baxendale, R. M. Myers, in 'Combinatorial Synthesis of Natural Product-Based Libraries', Ed. A. M. Boldi, CRC Press, Boca-Raton, Florida, USA, ISBN 0-8493-4000-4, 2006, Ch. 6, p 131.

[6] S. V. Ley, M. Ladlow, E. Vickerstaffe, 'Exploring Chemical Diversity for Drug Discovery', Eds P. A. Bartlett, M. Entzeroth, Royal Society of Chemistry, UK, ISBN-10:0-85404-842-1, 2006, p 3.

[7] S. V. Ley, R. M. Myers, 'The Chemical Theatre of Biological Systems', Eds M. G. Hicks, C. Kettner, Proceedings of the Beilstein-Institut Workshop, May 24th-28th 2004, Bozen, Italy, ISBN 3-8325-1019-2, p 1.

[8] I. R. Baxendale, A.-L. Lee, S. V. Ley, $J$. Chem. Soc., Perkin Trans. 1 2002, 1850.

[9] I. R. Baxendale, S. V. Ley, Bioorg. Med. Chem. Lett. 2000, 10, 1983.

[10] S. V. Ley, P. A. Procopiou, Org. Lett. 2002, 4, 3793.

[11] I. R. Baxendale, S. V. Ley, M. Nesi, C. Piutti, Tetrahedron 2002, 58, 6285.

[12] R. I. Storer, T. Takemoto, P. S. Jackson, D. S. Brown, I. R. Baxendale, S. V. Ley, Chem. Eur. J. 2004, 10, 2529.

[13] I. R. Baxendale, S. V. Ley, C. D. Smith, G. K. Tranmer, Org. Lett. 2006, 8, 5231.

[14] C. D. Smith, I. R. Baxendale, S. Lanners, J. J. Hayward, S. C. Smith, S. V. Ley, Org Biomol. Chem. 2007, 5, 1559.

[15] I. R. Baxendale, S. V. Ley, C. D. Smith, G. K. Tranmer, J. Chem. Soc., Chem. Commun. 2006, 4835.

[16] K. R. Knudsen, J. Holden, S. V. Ley, M. C. Ladlow, Adv. Syn. Cat. 2007, 349, 535.

[17] V. Franckevicius, K. R. Knudsen, M. C. Ladlow, D. A. Longbottom, S. V. Ley, Synlett 2006, 889.

[18] D. E. A. Brittain, S. V. Ley, Chemtracts 2005, 17, 620

[19] I. R. Baxendale, J. Deeley, C. M. GriffithsJones, S. V. Ley, S. Saaby, G. K. Tranmer, J. Chem. Soc., Chem. Commun. 2006, 2566.

[20] S. V. Ley, A. W. Thomas, H. Finch, J. Chem. Soc., Perkin Trans. 1 1999, 669.

[21] I. R. Baxendale, J. J. Hayward, S.V. Ley, Combinatorial Chemistry and High Throughput Screening 2007, 10, 802.

[22] C. D. Smith, I. R. Baxendale, G. K. Tranmer, M. Baumann, S. C. Smith, R. A. Lewthwaite, S. V. Ley, Org. Biomol. Chem. 2007, 5, 1562 .
[23] J. Siu, I. R. Baxendale, R. A. Lewthwaite, S. V. Ley, Org. Biomol. Chem. 2005, 3, 3140 .

[24] C. H. Hornung, M. R. Mackley, I. R. Baxendale, S. V. Ley, Org. Proc. Res. Dev. 2007, 11, 399 .

[25] N. Nikbin, M. Ladlow, S. V. Ley, Org. Proc. Res. Dev. 2007, 11, 458.

[26] F. Svec, J. M. J. Frechet, Science 1996, 273, 205.

[27] I. R. Baxendale, C. M. Griffiths-Jones, S. V. Ley, G. Tranmer, Chem. Eur. J. 2006 , 12, 4407.

[28] F. J. Iglesias-Sigüenza, C. J. Smith, I. R. Baxendale, S. V. Ley, Org. Biomol. Chem. 2007, 5, 2758 .

[29] A. M. Hafez, A. E. Taggi, H. Wack, W. J Drury III, T. Lectka, Org. Lett. 2000, 2 , 3963.

[30] G. Jas, A. Kirschning, Chem. Eur. J. 2003, 9, 5708.

[31] A. M. Hafez, A. E. Taggi, T. Lectka, Chem. Eur. J. 2002, 8, 4114.

[32] P. Hodge, Ind. Eng. Chem. Res. 2005, 44, 8455.

[33] W. Solodenko, G. Jas, V. Kung, A Kirschning, Synthesis 2007, 4, 583.

[34] M. Sands, S. J. Haswell, S. M. Kelly, V. Skelton, D. O. Morgan, P. Stryring, B. H. Warrington, Lab on a Chip 2001, 1, 64.

[35] D. Jönsson, B. H. Warrington, M. Ladlow, J. Comb. Chem. 2004, 6, 584.

[36] T. Kawaguchi, H. Miyata, K. Ataka, K. Mae, J.-I. Yoshida, Angew. Chem. Int. Ed. 2005, 44, 2413.

[37] E. R. Murphy, J. R. Martinelli, N. Zaborenko, S. L. Buchwald, K. F. Jensen, Angew. Chem Int. Ed. 2007, 46, 1734.

[38] B. Ahmed-Omer, J. C. Brandt, T. Wirth, Org. Biomol. Chem. 2007, 5, 733.

[39] M. S. Thomsen, P. Pölt, B. Nidetzky, $J$. Chem. Soc., Chem. Commun. 2007, 2527.

[40] M. I. Burguete, A. Cornejo, E. GarciaVerdugo, M. J. Gil, S. V. Luis, J. A. Mayoral, V. Martinez-Merino, M. Sokolova, J. Org. Chem. 2007, 72, 4344.

[41] H. Usutani, Y. Tomida, A. Nagaki, H. Okamoto, T. Nokami, J.-I. Yoshida, J. Am. Chem. Soc. 2007, 129, 3046.

[42] S. France, D. Bernstein, A. Weatherwax, T. Lectka, Org. Lett. 2005, 7, 3009.

[43] O. Flögel, J. D. C. Codée, D. Seebach, P. H. Seeberger, Angew. Chem. Int. Ed. 2006, 45,7000 .

[44] B. C. Hamper, E. Tesfu, Synlett 2007, 2257. 